\title{
Lipid-lowering therapy with PCSK9-inhibitors in the management of cardiovascular high-risk patients: Effectiveness, therapy adherence and safety in a real world cohort
}

\author{
Michael Saborowski*, Michael Dölle*, Michael P. Manns, \\ Holger Leitolf, Steffen Zender \\ Department for Gastroenterology, Hepatology and Endocrinology, \\ Hannover Medical School (MHH), Hannover, Germany
}

\begin{abstract}
Background: Proprotein convertase subtilisin/kexin type 9 (PCSK9)-inhibitors have shown great potential in efficient lipid lowering to achieve low-density lipoprotein-cholesterol (LDL-C) treatment goals. The aim of the study was too describe the clinical use of PCSK9-inhibitors and to investigate therapy adherence and safety outside of clinical trials.

Methods: Thirty-eight patients were treated with PSCK9-inhibitors. Patients were eligible for this therapy based on their individual cardiovascular risk and when all other available lipid-lowering regimen had failed. Every patient answered a questionnaire concerning medical history and relevant side effects and therapy adherence.

Results: Conventional therapy reduced patient LDL-C levels by about 38\%. However, in 26 of the 38 patients, $L D L-C$ treatment goals were not fulfilled because patients did not tolerate further dose escalation due to side effects. Using a PCSK9 inhibitor, LDL-C levels were reduced by another $54 \%$ and $42 \%$ of patients reaching treatment goals. The results show that most patients still require concomitant therapy to reach $L D L-C$ target levels. Three patients required dose reduction or change of the PCSK9 inhibitor. $16 \%$ did not inject the PCSK9 inhibitor regularly.

Conclusions: Only a minority of patients reached the recommended LDL-C goals. PCSK9-inhibitors were generally well tolerated. Despite low rates of reported side effects, therapy adherence was incomplete, with 6 patients not injecting PCSK9-inhibitors on a regular basis. In-depth information about the medication and close supervision is advisable. PCSK9 inhibitors have shown great potential in aggressive lipid lowering therapy, but basic therapy is still required in most cases. Close supervision is recommended to improve therapy adherence. (Cardiol J 2018; 25, 1: 32-41)
\end{abstract}

Key words: cardiovascular risk, lipid lowering therapy, PCSK9-inhibitors, prevention, therapy adherence

\section{Introduction}

\section{Cardiovascular disease burden}

Cardiovascular disease (CVD) remains the most common cause of death world-wide. The Global Burden of Disease Study, published in 2013, estimates the total number of coronary artery disease (CAD)-related deaths at 17.3 million per year, accounting for $31.5 \%$ of all deaths and for $45 \%$ of all non-communicable deaths [1]. According to 'Cardiovascular disease in Europe: epidemiological update 2016', CVD causes over 4 million deaths

Address for correspondence: Dr. Steffen Zender, Department for Gastroenterology, Hepatology and Endocrinology, Hannover Medical School (MHH), Carl-Neuberg-Straße 1, 30625 Hannover, Germany, e-mail: zender.steffen@mh-hannover.de

Received: 13.08.2017 Accepted: 01.11.2017

*Equal contribution. 
per year, 1.4 million deaths occurring in patients younger than 75 years. The number of cardiovascular deaths in women (2.2 million or 49\%) is slightly higher than in men (1.8 million or 40\%) [2].

\section{Low-density lipoprotein cholesterol as} a causal factor for cardiovascular disease

The INTERHEART study (2004) characterized the effect of potentially modifiable risks for myocardial infarction in 52 countries [3]. Factors elevating cardiovascular risk were current or previous smoking, diabetes mellitus, elevated lowdensity lipoprotein cholesterol (LDL-C), hypertension, abdominal obesity, irregular consumption of fruit and vegetables, lack of moderate alcohol consumption and increased psychosocial stress. The presence of multiple risk factors led to an increased global cardiovascular risk and to the concept of a multi-modular intervention strategy to effectively reduce the risk for myocardial infarction.

Numerous studies over the last decades have demonstrated a causal relationship between LDL-C concentration and manifestation or progression of atherosclerosis and CVD. Experimental studies on the initiation of atherosclerosis, results from molecular and Mendelian randomization studies [4], and studies of familial hypercholesterolemia [5] firmly support this concept. Pharmacological inhibition of hepatic cholesterol synthesis or intestinal cholesterol resorption depletes intracellular hepatic cholesterol, leading to increased expression of the hepatic LDL-C receptor and increased LDL-C plasma clearance. Large randomized controlled studies using statins as inhibitors of HMG-CoA-reductase [6], and ezetimibe as inhibitors of NPLC1L1 [7] unanimously demonstrated a decrease of cardiovascular events. The long-term follow-up of LDL-C lowering therapy shows that cardiovascular outcome even after termination of the active intervention phase is improved (legacy effect) [8].

A recent review summarizing the efficacy and safety of statin therapy estimated that a reduction of LDL-C by $1 \mathrm{mmol} / \mathrm{L}$ results in about a $25 \%$ reduction in the rate of vascular events (defined as coronary death, myocardial infarction, stroke and coronary revascularization). Furthermore, a reduction by $2 \mathrm{mmol} / \mathrm{L}$ reduces the rate of cardiovascular events of approximately $45 \%$. In other words, treating 10,000 high-risk patients over a period of 5 years would result in about 1,000 fewer vascular events [9]. Simultaneously, no effects of statin therapy on non-vascular events and death were found.

\section{ESC/EAS Guidelines 2016}

In 2016, the European Society of Cardiology (ESC) together with the European Atherosclerosis Society (EAS) published comprehensive guidelines for the prevention of CVD and for the treatment of dyslipidemia [10, 11]. These guidelines rely on an individual estimation of global cardiovascular risk, and grade the recommended intensity of LDL-C lowering therapy according to four distinct risk categories. The very high risk group includes patients with documented CVD or cerebrovascular disease, aortic aneurysm, peripheral artery disease or significant plaque burden, patients with diabetes mellitus with target organ damage or an additional major risk factor and severe chronic kidney disease (defined as eGFR $<30 \mathrm{~mL} / \mathrm{min} / 1.73 \mathrm{~m}^{2}$ ). The high risk group includes patients with elevated single risk factors such as familial hypercholesterolemia, diabetes mellitus without additional major risk factors, and moderate chronic kidney disease (eGFR $30-59 \mathrm{~mL} / \mathrm{min} / 1.73 \mathrm{~m}^{2}$ ). The guidelines recommend a LDL-C goal $<70 \mathrm{mg} / \mathrm{dL}$ or a reduction of at least $50 \%$ if the baseline is between 70 and $135 \mathrm{mg} / \mathrm{dL}$ for the very high-risk group, and a LDL-C goal $<100 \mathrm{mg} / \mathrm{dL}$ or a reduction of at least $50 \%$ if the baseline is between 100 and $200 \mathrm{mg} / \mathrm{dL}$ for the high-risk group.

\section{PCSK9 inhibitors as a new class \\ of cholesterol lowering drugs}

Proprotein convertase subtilisin/kexin type 9 (PCSK9) is a serine protease involved in cholesterol homeostasis. PCSK9 binds to the complex LDL-receptor inducing intracellular degradation, thereby reducing serum LDL clearance. The subcutaneously injected monoclonal antibodies directed against PCSK9, alirocumab and evolocumab are approved by The Food and Drug Administration and European Medicines Agency for the treatment of hypercholesterolemia in patients with intolerance or inadequate response to statins, especially for secondary prevention in a very high-risk situation or in the case of familial hypercholesterolemia. PCSK9 inhibition enables more efficient hepatic uptake of LDL and in most cases, decreases serum LDL levels by $>50 \%$, showing that PCSK9 inhibition is an innovative therapeutic approach to improve control of elevated LDL-C levels.

In October 2016, the ESC and the EAS Task Force published a consensus statement discussing the appropriate clinical use of PCSK9 antibodies in patients at very high cardiovascular risk who have substantially elevated LDL-C levels despite maximal statin/ezetimibe therapy [12]. 


\section{Methods}

Thirty-eight patients from the lipid outpatient clinic of the Hanover Medical School that had been treated with a PCSK9 inhibitor (alirocumab 75/150 mg or evolocumab $140 \mathrm{mg}$ ) until present were retrospectively analyzed. Patients were classified as high risk or very high risk according to ESC guidelines of dyslipidemia, 2016 [11]. The LDL-C goals were as follows:

- high risk - LDL-C < $100 \mathrm{mg} / \mathrm{dL}$;

- very high risk - LDL-C $<70 \mathrm{mg} / \mathrm{dL}$.

Until 2016, PCSK9 treatment was begun if patients did not reach their LDL-C treatment goal under maximum tolerated lipid lowering therapy (e.g. statin, ezetimibe, colesevelam) or did not tolerate dose escalation due to side effects. After publication of the consensus statement from the ESC/EAS [12] we initiated PCSK9 treatment in very high-risk patients according to ESC guidelines [11] exhibiting LDL-C level $>140 \mathrm{mg} / \mathrm{dL}$ or in patients suffering from a progredient CVD who had LDL-C levels above $100 \mathrm{mg} / \mathrm{dL}$. Patients in a high risk situation without evidence of CVD were also treated with a PCSK 9 inhibitor if they had LDL-C levels above $175 \mathrm{mg} / \mathrm{dL}$ and further cardiovascular risk factors.

Genetic testing was performed sequentially:

1. Sequencing of the entire LDL receptor;

2. If 1 . was negative, sequencing of ApoB100 gene;

3. If all of the above were negative, sequencing of the PCSK9 gene.

Positive family history was defined as a premature CAD or cerebrovascular event in women $<60$ years and men $<55$ years of age (in patients or first-degree relatives).

The Dutch Lipid Clinic Network was used for diagnostic criteria for familial hypercholesterinemia $[5,13,14]$.

Lipid electrophoresis was performed using Hydragel 54 LDL/HDL CHOL Direct (Sebia). Lipoprotein(a) $[\mathrm{Lp}(\mathrm{a})]$ measurement was performed with Tina-quant ${ }^{\circledR}$ Lipoprotein (a) Gen. 2 (Cobas).

Relevant $\mathrm{Lp}$ (a) elevation was defined as $\mathrm{Lp}$ (a) levels $>60 \mathrm{mg} / \mathrm{dL}$ or $150 \mathrm{nmol} / \mathrm{L}[15,16]$.

All patients were asked to fill out a questionnaire on a voluntary basis concerning their history of CVD and experience with PCSK9 inhibitor treatment (Supplemental Figure 1). Demographic characteristics, laboratory results, and results from genetic testing for mutations in the LDL-receptor, ApoB gene or PCSK9 gene were extracted from our lipid outpatient clinic database. Statistical analysis was performed with Microsoft Excel ${ }^{\circledast}$ using t-test without pretesting $[17,18]$. SDAPS 1.9 .4 was used to create and analyze the questionnaires. This study was approved by the local ethics committee (Ethics committee Hannover Medical School \# 3471-2017).

\section{Results}

As of June 201738 high risk and very high-risk cardiovascular patients were treated with PCSK9 inhibitors, either alirocumab $(n=19)$ or evolocumab $(n=19) .18$ patients were male and 20 were female (Table 1). One patient was lost to follow up after initiation of PCSK9 therapy. The average age was 59.7 years. The baseline LDL-C level from all patients was about $260 \mathrm{mg} / \mathrm{dL}$. Five patients had both increased LDL-C levels and significantly increased $\mathrm{Lp}$ (a) elevation (> $150 \mathrm{nmol} / \mathrm{L}$ ). The 2 patients of the high-risk category had a target range for LDL-C of $<100 \mathrm{mg} / \mathrm{dL}$. The 36 very high-risk patients had a target range for LDL-C of $<70 \mathrm{mg} / \mathrm{dL}$. During first contact with patients we were able to work out a positive family history of myocardial infarction or ischemic stroke in about half of the patients. 23 patients gave consent for genetic testing for familial hypercholesterinemia, of which 17 had genetic alterations in the LDL receptor. Six of the patients did not exhibit any genetic abnormalities.

After admission to the lipid outpatient clinic, patients were initially treated with statins, colesevelam, or ezetimibe-based therapies. Only 5 patients out of 38 patients were treated with maximum doses of (atorvastatin $80 \mathrm{mg}$, ezetimibe $10 \mathrm{mg}$ or colesevelam $625 \mathrm{mg} 6 \times$ daily) (Table 2). Even after changing the initial medication to other lipidlowering drugs, only 12 patients had a combination therapy of ezetimibe and a statin in their respective maximum dosage. In the remaining patients, escalation to maximum dose was not achieved due to side effects such as statin-associated muscle symptoms or other symptoms such as articular pain and/or dizziness. In total, only 14 of 38 patients reached the allowed maximum dosage of the newly introduced statin (for example atorvastatin $80 \mathrm{mg}$, fluvastatin $80 \mathrm{mg}$, simvastatin $40 \mathrm{mg}$, rosuvastatin $40 \mathrm{mg}$ or pravastatin $40 \mathrm{mg}$ ). Five patients were on a therapy without any statin (such as ezetimibe or colesevelam). Utilizing different combinations of these drugs, average LDL-C levels were able to be reduced by about $38 \%$ (Fig. 1)

By adding a PCSK9 inhibitor the LDL-C levels were able to be reduced by another $54 \%$ (Fig. 1). In 
Table 1. Patient characteristics: 38 patients (18 male and 20 female) from the documented outpatient lipid clinic were retrospectively analyzed. 36 out of 38 patients had documented coronary artery disease (CAD). 22/38 patients had a family history of CAD and 5/38 patients an elevation of lipoprotein(a) [Lp(a)]. Median age was 59.7 years.

\begin{tabular}{|c|c|c|c|c|c|c|}
\hline Patient & Sex & Age & $\mathbf{F H}$ & Disease & Lp(a) [nmol/L (mg/dL)] & Elevation \\
\hline 1 & M & 42 & Pos. & 3-vessel CAD, MI, stenting & $24(10)$ & No \\
\hline 2 & $\mathrm{~F}$ & 65 & Neg. & AC plaque, aortic sclerosis & $288(120)$ & Yes \\
\hline 3 & $\mathrm{~F}$ & 76 & Pos. & $\mathrm{ACl}$ plaque & $12(5)$ & No \\
\hline 4 & $\mathrm{~F}$ & 70 & Neg. & 2-vessel CAD, PAD, stenting & $143(60)$ & No \\
\hline 5 & $\mathrm{~F}$ & 70 & Neg. & 3-vessel CAD, MI, stenting & NA & NA \\
\hline 6 & M & 56 & Neg. & MI & $17(7)$ & No \\
\hline 7 & $\mathrm{~F}$ & 72 & Neg. & $\mathrm{ACl}$ stenosis, 3-vessel CAD, CABG, PAD & $137(57)$ & No \\
\hline 8 & $\mathrm{~F}$ & 52 & Pos. & AC plaque, aortic sclerosis & $46(19)$ & No \\
\hline 9 & $\mathrm{~F}$ & 61 & Pos. & 3-vessel CAD, CABG & $52(22)$ & No \\
\hline 10 & M & 47 & Neg. & 3-vessel CAD, PAD & $312(130)$ & Yes \\
\hline 11 & M & 53 & Neg. & 2-vessel CAD, AC plaque, CABG & $60(25)$ & No \\
\hline 12 & M & 60 & Pos. & Aortic sclerosis & $62(26)$ & No \\
\hline 13 & M & 55 & Neg. & 3-vessel CAD, CABG & $20(8)$ & No \\
\hline 14 & M & 79 & Pos. & $C A D$, ischemic stroke & $64(27)$ & No \\
\hline 15 & M & 60 & Pos. & AC plaque, aortic sclerosis & $7(3)$ & No \\
\hline 16 & $\mathrm{~F}$ & 69 & N.a. & AC plaque, aortic sclerosis & $19(8)$ & No \\
\hline 17 & $\mathrm{~F}$ & 71 & Pos. & 3-vessel CAD, CABG & $20(8)$ & No \\
\hline 18 & M & 57 & Neg. & $C A D, C A B G$ & $14(6)$ & No \\
\hline 19 & $\mathrm{~F}$ & 61 & Pos. & AC plaque, aortic sclerosis & $42(17)$ & No \\
\hline 20 & M & 36 & N.a. & AC plaque, aortic sclerosis & $65(27)$ & No \\
\hline 21 & $\mathrm{~F}$ & 64 & Neg. & AC plaque, aortic sclerosis & $7(3)$ & No \\
\hline 22 & $\mathrm{~F}$ & 67 & Pos. & 3-vessel CAD, stenting & $20(8)$ & No \\
\hline 23 & M & 67 & Neg. & 2-vessel CAD, AC plaque, CABG & $36(15)$ & No \\
\hline 24 & M & 50 & Pos. & 3-vessel CAD, MI, resuscitation, stenting & $20(8)$ & No \\
\hline 25 & M & 70 & Neg. & 3-vessel CAD, MI & $58(24)$ & No \\
\hline 26 & $\mathrm{~F}$ & 44 & Pos. & Primary prevention & $118(49)$ & No \\
\hline 27 & $\mathrm{~F}$ & 70 & Pos. & Aortic sclerosis & $163(68)$ & Yes \\
\hline 28 & M & 63 & Pos. & 3-vessel CAD, MI, stroke & $19(8)$ & No \\
\hline 29 & $\mathrm{~F}$ & 42 & Pos. & Primary prevention & $27(11)$ & No \\
\hline 30 & M & 66 & Neg. & Aortic sclerosis & $19(8)$ & No \\
\hline 31 & M & 55 & Pos. & 2-vessel CAD, MI & $20(8)$ & No \\
\hline 32 & M & 44 & Pos. & 2-vessel CAD, MI, stenting & $255(106)$ & Yes \\
\hline 33 & $\mathrm{~F}$ & 60 & Neg. & AC plaque, stroke & NA & NA \\
\hline 34 & $\mathrm{~F}$ & 42 & Pos. & AC plaque & $259(108)$ & Yes \\
\hline 35 & $\mathrm{~F}$ & 53 & Pos. & 1-vessel CAD, MI & $58(24)$ & No \\
\hline 36 & $\mathrm{~F}$ & 70 & Pos. & 3-vessel CAD, MI & $142(59)$ & No \\
\hline 37 & M & 48 & Pos. & Aortic sclerosis & $144(60)$ & No \\
\hline 38 & M & 39 & Pos. & 3-vessel CAD, MI & $<20(<8)$ & No \\
\hline
\end{tabular}

$\mathrm{AC}$ - arteria carotis; $\mathrm{ACl}$ - arteria carotis interna; $\mathrm{CABG}$ - coronary artery bypass grafting; $\mathrm{F}$ - female; $\mathrm{M}$ - male; NA - not available; $\mathrm{MI}$ - myocardial infarction; neg. - negative; PAD — peripheral artery disease; pos. — positive

total, conventional therapy and PCSK9 inhibitors in combination led to a reduction of LDL-C levels of about $70 \%$ when compared to the therapy-naïve situation. Despite this potent reduction, only $1(50 \%)$ patient of the high risk group and $15(42 \%)$ patients of the very high risk group reached target 
Table 2. Low density lipoprotein cholesterol (LDL-C) levels under different lipid lowering treatment regimens.

\begin{tabular}{|c|c|c|c|c|c|c|c|c|c|}
\hline No. & $\begin{array}{l}\text { Previous } \\
\text { medication }\end{array}$ & $\begin{array}{l}\text { Escalation } \\
\text { to PCSK9 }\end{array}$ & $\begin{array}{l}\text { LDL-0 } \\
{[\mathrm{mg} / \mathrm{dL}]}\end{array}$ & $\begin{array}{l}\text { LDL-1 } \\
{[\mathrm{mg} / \mathrm{dL}]}\end{array}$ & $\begin{array}{l}\text { Red. } \\
{[\%]}\end{array}$ & $\begin{array}{l}\text { LDL-2 } \\
{[\mathrm{mg} / \mathrm{dL}]}\end{array}$ & $\begin{array}{l}\text { Red. } \\
{[\%]}\end{array}$ & $\begin{array}{l}\text { PCSK9 } \\
\text { inhibitor }\end{array}$ & $\begin{array}{l}\text { LDL-C } \\
\text { goal }\end{array}$ \\
\hline 1 & Naïve & $\begin{array}{c}\text { Statin } \\
\text { intolerance }\end{array}$ & 197 & 197 & 0 & 91 & 54 & $\begin{array}{l}\text { Alirocumab } \\
75 \mathrm{mg}\end{array}$ & No \\
\hline 2 & Atorvastatin $60 \mathrm{mg}$ & $\begin{array}{l}\text { Intolerance to } \\
\text { higher dose, } \\
\text { ezetimibe } \\
\text { intolerance }\end{array}$ & 277 & 209 & 25 & 153 & 27 & $\begin{array}{l}\text { Alirocumab } \\
150 \mathrm{mg}\end{array}$ & No \\
\hline 3 & $\begin{array}{c}\text { Atorvastatin } 20 \mathrm{mg} \\
\text { colesevelam }\end{array}$ & $\begin{array}{l}\text { Intolerance to } \\
\text { higher dose }\end{array}$ & 180 & 163 & 9 & 124 & 24 & $\begin{array}{l}\text { Evolocumab } \\
140 \mathrm{mg}\end{array}$ & No \\
\hline 4 & Fluvastatin $80 \mathrm{mg}$ & $\begin{array}{c}\text { Statin intolerance, } \\
\text { ezetimibe } \\
\text { intolerance }\end{array}$ & 204 & 110 & 46 & 20 & 81 & $\begin{array}{l}\text { Alirocumab } \\
75 \mathrm{mg}\end{array}$ & Yes \\
\hline 5 & $\begin{array}{l}\text { Pravastatin } 40 \mathrm{mg} \text {, } \\
\text { ezetimibe } 10 \mathrm{mg}\end{array}$ & Statin intolerance & 358 & 268 & 25 & 79 & 70 & $\begin{array}{l}\text { Alirocumab } \\
150 \mathrm{mg}\end{array}$ & No \\
\hline 6 & $\begin{array}{l}\text { Atorvastatin } 40 \mathrm{mg} \text {, } \\
\text { ezetimibe } 10 \mathrm{mg}\end{array}$ & $\begin{array}{l}\text { Intolerance to } \\
\text { higher dose }\end{array}$ & NA & 136 & NA & 50 & 63 & $\begin{array}{l}\text { Evolocumab } \\
140 \mathrm{mg}\end{array}$ & Yes \\
\hline 7 & $\begin{array}{c}\text { Atorvastatin } 40 \mathrm{mg} \text {, } \\
\text { colesevelam }\end{array}$ & $\begin{array}{l}\text { Intolerance to } \\
\text { higher dose }\end{array}$ & 233 & 96 & 59 & 63 & 34 & $\begin{array}{l}\text { Alirocumab } \\
75 \mathrm{mg}\end{array}$ & Yes \\
\hline 8 & $\begin{array}{l}\text { Fluvastatin } 80 \mathrm{mg} \text {, } \\
\text { ezetimibe } 10 \mathrm{mg} \text {, } \\
\text { colesevelam }\end{array}$ & $\begin{array}{c}\text { Statin } \\
\text { intolerance }\end{array}$ & 299 & 156 & 48 & 79 & 49 & $\begin{array}{l}\text { Evolocumab } \\
140 \mathrm{mg}\end{array}$ & No \\
\hline 9 & $\begin{array}{l}\text { Atorvastatin } 80 \mathrm{mg} \text {, } \\
\text { ezetimibe } 10 \mathrm{mg} \text {, } \\
\text { colesevelam }\end{array}$ & $\begin{array}{l}\text { Maximum dose, } \\
\text { missed target } \\
\text { range }\end{array}$ & 388 & 122 & 69 & 32 & 53 & $\begin{array}{l}\text { Evolocumab } \\
140 \mathrm{mg}\end{array}$ & Yes \\
\hline 10 & $\begin{array}{l}\text { Rosuvastatin } 20 \mathrm{mg} \text {, } \\
\text { ezetimibe } 10 \mathrm{mg}\end{array}$ & $\begin{array}{c}\text { Statin } \\
\text { intolerance }\end{array}$ & 245 & 112 & 54 & 34 & 70 & $\begin{array}{l}\text { Evolocumab } \\
140 \mathrm{mg}\end{array}$ & Yes \\
\hline 11 & $\begin{array}{l}\text { Atorvastatin } 80 \mathrm{mg} \text {, } \\
\text { ezetimibe } 10 \mathrm{mg} \text {, } \\
\text { colesevelam }\end{array}$ & $\begin{array}{l}\text { Maximum dose, } \\
\text { missed target } \\
\text { range }\end{array}$ & NA & 120 & NA & 21 & 83 & $\begin{array}{l}\text { Alirocumab } \\
75 \mathrm{mg}\end{array}$ & Yes \\
\hline 12 & $\begin{array}{l}\text { Atorvastatin } 80 \mathrm{mg} \text {, } \\
\text { ezetimibe } 10 \mathrm{mg} \text {, } \\
\text { colesevelam }\end{array}$ & $\begin{array}{l}\text { Maximum dose, } \\
\text { missed target } \\
\text { range }\end{array}$ & 258 & 154 & 40 & 35 & 87 & $\begin{array}{l}\text { Alirocumab } \\
150 \mathrm{mg}\end{array}$ & Yes \\
\hline 13 & Ezetimibe $10 \mathrm{mg}$ & $\begin{array}{c}\text { Statin } \\
\text { intolerance }\end{array}$ & NA & 150 & NA & 80 & 47 & $\begin{array}{l}\text { Alirocumab } \\
150 \mathrm{mg}\end{array}$ & No \\
\hline 14 & Naïve & $\begin{array}{c}\text { Statin } \\
\text { intolerance, } \\
\text { ezetimibe } \\
\text { intolerance }\end{array}$ & NA & 141 & NA & 80 & 43 & $\begin{array}{l}\text { Alirocumab } \\
75 \mathrm{mg}\end{array}$ & No \\
\hline 15 & $\begin{array}{l}\text { Atovastatin } 40 \mathrm{mg} \text {, } \\
\text { ezetimibe } 10 \mathrm{mg}\end{array}$ & $\begin{array}{l}\text { Intolerance to } \\
\text { higher dose }\end{array}$ & 276 & 144 & 48 & 34 & 76 & $\begin{array}{l}\text { Evolocumab } \\
140 \mathrm{mg}\end{array}$ & Yes \\
\hline 16 & Naïve & $\begin{array}{l}\text { Statin } \\
\text { intolerance, } \\
\text { ezetimibe } \\
\text { intolerance }\end{array}$ & 264 & 264 & 0 & 114 & 57 & $\begin{array}{l}\text { Alirocumab } \\
150 \mathrm{mg}\end{array}$ & No \\
\hline 17 & Naïve & $\begin{array}{l}\text { Statin } \\
\text { intolerance, } \\
\text { ezetimibe } \\
\text { intolerance }\end{array}$ & 195 & 195 & 0 & 121 & 38 & $\begin{array}{l}\text { Alirocumab } \\
75 \mathrm{mg}\end{array}$ & No \\
\hline 18 & Colesevelam & $\begin{array}{l}\text { Statin } \\
\text { intolerance, } \\
\text { ezetimibe } \\
\text { intolerance }\end{array}$ & 198 & 154 & 22 & 64 & 59 & $\begin{array}{l}\text { Evolocumab } \\
140 \mathrm{mg}\end{array}$ & Yes \\
\hline 19 & $\begin{array}{l}\text { Rosuvastatin } 40 \mathrm{mg} \text {, } \\
\text { ezetimibe } 10 \mathrm{mg}\end{array}$ & $\begin{array}{l}\text { Maximum dose, } \\
\text { missed target } \\
\text { range }\end{array}$ & n.a. & 168 & NA & 86 & 48 & $\begin{array}{l}\text { Alirocumab } \\
150 \mathrm{mg}\end{array}$ & No \\
\hline
\end{tabular}


Table 2 (cont.). Low density lipoprotein cholesterol (LDL-C) levels under different lipid lowering treatment regimens.

\begin{tabular}{|c|c|c|c|c|c|c|c|c|c|}
\hline No. & $\begin{array}{l}\text { Previous } \\
\text { medication }\end{array}$ & $\begin{array}{l}\text { Escalation } \\
\text { to PCSK9 }\end{array}$ & $\begin{array}{l}\text { LDL-0 } \\
{[\mathrm{mg} / \mathrm{dL}]}\end{array}$ & $\begin{array}{l}\text { LDL-1 } \\
{[\mathrm{mg} / \mathrm{dL}]}\end{array}$ & $\begin{array}{l}\text { Red. } \\
{[\%]}\end{array}$ & $\begin{array}{l}\text { LDL-2 } \\
{[\mathrm{mg} / \mathrm{dL}]}\end{array}$ & $\begin{array}{l}\text { Red. } \\
{[\%]}\end{array}$ & $\begin{array}{l}\text { PCSK9 } \\
\text { inhibitor }\end{array}$ & $\begin{array}{l}\text { LDL-C } \\
\text { goal }\end{array}$ \\
\hline 20 & $\begin{array}{l}\text { Atorvastatin } 80 \mathrm{mg} \text {, } \\
\text { ezetimibe } 10 \mathrm{mg}, \\
\text { colesevelam }\end{array}$ & $\begin{array}{l}\text { Maximum dose, } \\
\text { missed target } \\
\text { range }\end{array}$ & 520 & 200 & 61 & 102 & 49 & $\begin{array}{l}\text { Evolocumab } \\
140 \mathrm{mg}\end{array}$ & No \\
\hline 21 & $\begin{array}{l}\text { Colesevelam, } \\
\text { ezetimibe } 10 \mathrm{mg}\end{array}$ & $\begin{array}{c}\text { Statin } \\
\text { intolerance }\end{array}$ & 279 & 189 & 32 & 85 & 55 & $\begin{array}{l}\text { Evolocumab } \\
140 \mathrm{mg}\end{array}$ & No \\
\hline 22 & Naïve & $\begin{array}{c}\text { Statin } \\
\text { intolerance }\end{array}$ & NA & 126 & NA & 39 & 69 & $\begin{array}{l}\text { Alirocumab } \\
150 \mathrm{mg}\end{array}$ & Yes \\
\hline 23 & $\begin{array}{l}\text { Colesevelam, } \\
\text { ezetimibe } 10 \mathrm{mg}\end{array}$ & $\begin{array}{c}\text { Statin } \\
\text { intolerance }\end{array}$ & 212 & 120 & 43 & 81 & 32 & $\begin{array}{l}\text { Evolocumab } \\
140 \mathrm{mg}\end{array}$ & No \\
\hline 24 & Naïve & $\begin{array}{c}\text { Statin intolerance, } \\
\text { ezetimibe } \\
\text { intolerance }\end{array}$ & NA & 77 & NA & 38 & 51 & $\begin{array}{l}\text { Evolocumab } \\
140 \mathrm{mg}\end{array}$ & Yes \\
\hline 25 & Naïve & $\begin{array}{c}\text { Statin intolerance, } \\
\text { ezetimibe } \\
\text { intolerance }\end{array}$ & 207 & 196 & 5,3 & 114 & 42 & $\begin{array}{l}\text { Alirocumab } \\
150 \mathrm{mg}\end{array}$ & No \\
\hline 26 & $\begin{array}{l}\text { Simvastatin } 40 \mathrm{mg} \text {, } \\
\text { ezetimibe } 10 \mathrm{mg}\end{array}$ & $\begin{array}{l}\text { Maximum dose, } \\
\text { missed target } \\
\text { range }\end{array}$ & 311 & 167 & 46 & 86 & 53 & $\begin{array}{l}\text { Alirocumab } \\
150 \mathrm{mg}\end{array}$ & Yes \\
\hline 27 & $\begin{array}{l}\text { Atorvastatin } 40 \mathrm{mg}, \\
\text { ezetimibe } 10 \mathrm{mg}\end{array}$ & $\begin{array}{l}\text { Maximum dose, } \\
\text { missed target } \\
\text { range }\end{array}$ & NA & 127 & NA & 51 & 60 & $\begin{array}{l}\text { Alirocumab } \\
75 \mathrm{mg}\end{array}$ & Yes \\
\hline 28 & $\begin{array}{l}\text { Simvastatin } 40 \mathrm{mg} \text {, } \\
\text { ezetimibe } 10 \mathrm{mg}\end{array}$ & $\begin{array}{l}\text { Maximum dose, } \\
\text { missed target } \\
\text { range }\end{array}$ & 287 & 178 & 38 & 82 & 54 & $\begin{array}{l}\text { Evolocumab } \\
140 \mathrm{mg}\end{array}$ & No \\
\hline 29 & Naïve & $\begin{array}{c}\text { Statin } \\
\text { intolerance }\end{array}$ & 324 & 260 & 20 & 231 & 12 & $\begin{array}{l}\text { Evolocumab } \\
140 \mathrm{mg}\end{array}$ & No \\
\hline 30 & Ezetimibe $10 \mathrm{mg}$ & $\begin{array}{c}\text { Statin } \\
\text { intolerance }\end{array}$ & 251 & 251 & 0 & 99 & 60 & $\begin{array}{l}\text { Alirocumab } \\
75 \mathrm{mg}\end{array}$ & No \\
\hline 31 & $\begin{array}{l}\text { Atorvastatin } 80 \mathrm{mg} \text {, } \\
\text { Ezetimibe } 10 \mathrm{mg}\end{array}$ & $\begin{array}{l}\text { Maximum dose, } \\
\text { missed target } \\
\text { range }\end{array}$ & NA & 152 & NA & & & $\begin{array}{l}\text { Evolocumab } \\
140 \mathrm{mg}\end{array}$ & NA \\
\hline 32 & $\begin{array}{l}\text { Atorvastatin } 40 \mathrm{mg} \text {, } \\
\text { Ezetimibe } 10 \mathrm{mg}\end{array}$ & $\begin{array}{l}\text { Intolerance to } \\
\text { higher dose }\end{array}$ & 160 & 115 & 28.3 & 54 & 53 & $\begin{array}{l}\text { Evolocumab } \\
140 \mathrm{mg}\end{array}$ & Yes \\
\hline 33 & Rosuvastatin $10 \mathrm{mg}$ & $\begin{array}{l}\text { Intolerance to } \\
\text { higher dose }\end{array}$ & NA & 155 & NA & 81 & 48 & $\begin{array}{l}\text { Alirocumab } \\
150 \mathrm{mg}\end{array}$ & No \\
\hline 34 & Atorvastatin 80 mg & $\begin{array}{l}\text { Ezetimibe } \\
\text { intolerance }\end{array}$ & 210 & 155 & 26 & 81 & 48 & $\begin{array}{l}\text { Alirocumab } \\
75 \mathrm{mg}\end{array}$ & No \\
\hline 35 & $\begin{array}{l}\text { Atorvastatin } 80 \mathrm{mg} \text {, } \\
\text { ezetimibe } 10 \mathrm{mg}, \\
\text { colesevelam }\end{array}$ & $\begin{array}{l}\text { Maximum dose, } \\
\text { missed target } \\
\text { range }\end{array}$ & 249 & 149 & 40 & 58 & 61 & $\begin{array}{l}\text { Evolocumab } \\
140 \mathrm{mg}\end{array}$ & Yes \\
\hline 36 & Naïve & $\begin{array}{c}\text { Statin } \\
\text { intolerance }\end{array}$ & 208 & 208 & NA & 177 & 15 & $\begin{array}{l}\text { Evolocumab } \\
140 \mathrm{mg}\end{array}$ & No \\
\hline 37 & $\begin{array}{c}\text { Simvastatin } 40 \mathrm{mg} \\
\text { ezetimibe } 10\end{array}$ & $\begin{array}{l}\text { Maximum dose, } \\
\text { missed target } \\
\text { range }\end{array}$ & 348 & 191 & 45 & 92 & 51 & $\begin{array}{l}\text { Evolocumab } \\
140 \mathrm{mg}\end{array}$ & No \\
\hline 38 & Atorvastatin $40 \mathrm{mg}$ & $\begin{array}{l}\text { Intolerance to } \\
\text { higher dose }\end{array}$ & 168 & 110 & 35 & 19 & 82 & $\begin{array}{l}\text { Evolocumab } \\
140 \mathrm{mg}\end{array}$ & Yes \\
\hline
\end{tabular}

LDL-0 - LDL-C levels before start of a lipid lowering therapy; LDL-1 - LDL-C values after reaching maximum tolerated dose with statins, ezetimibe or colesevelam; LDL-2 — LDL-C after addition of a PCSK9 inhibitor; NA - not available 


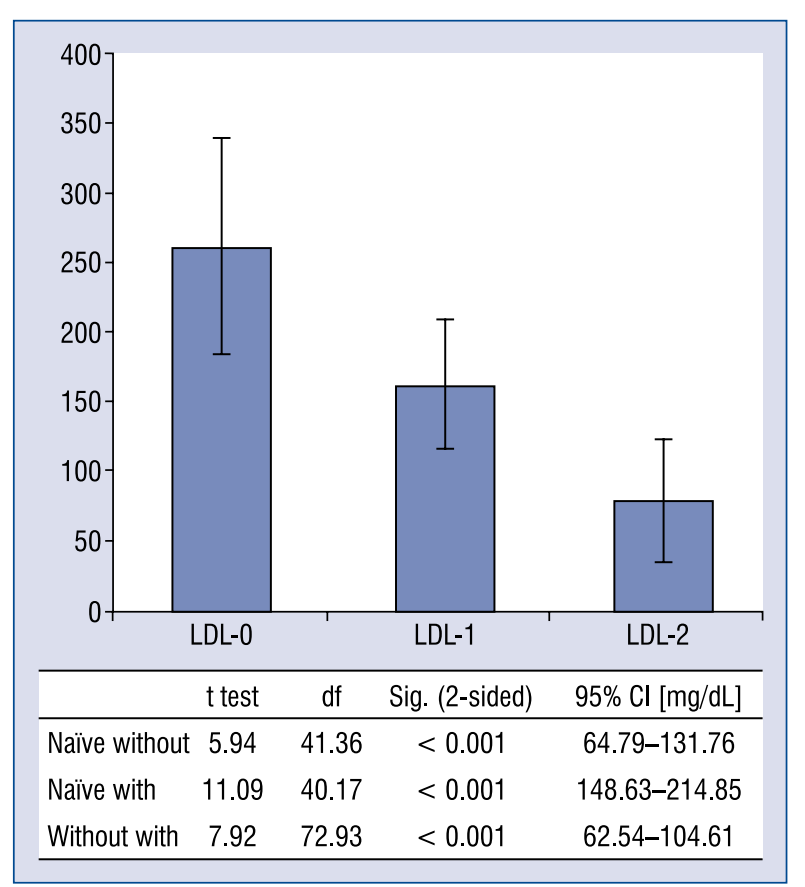

Figure 1. Average low density lipoprotein cholesterol (LDL-C) levels under different lipid lowering treatment regimens; LDL-0 — LDL-C levels before begin of a lipid lowering therapy (naïve); LDL-1 - LDL-C values after reaching maximum tolerated dose with statins, ezetimibe or colesevelam (without PCSK9); LDL-2 LDL-C after addition of a PCSK9 inhibitor (with); df degree of freedom; $\mathrm{Cl}$ - confidence interval.

LDL-C levels. In addition, $\mathrm{Lp}(\mathrm{a})$ levels after initiation of PCSK9 inhibitor treatment were also measured in patients with significantly elevated $L p(a)$ $(>150 \mathrm{nmol} / \mathrm{L})$. Four out of these 5 patients showed a reduction of $\mathrm{Lp}$ (a) levels (average 25.4\%) upon PCSK9 inhibitor treatment. One patient exhibited a $31.3 \%$ increase of $\mathrm{Lp}(\mathrm{a})$ (Table 3 ).

Six patients, all female, reported side effects of PCSK9 inhibitor treatment. All of these patients had also experienced side effects under their previous lipid-lowering regimen (e.g. mus- cle pain). Three of these patients continued their medication despite these side effects. One female patient reacted with severe hypotension after injection of $140 \mathrm{mg}$ evolocumab. After the second injection hypotension occurred again and led to hospitalization. Side effects, risks and benefits of this medication were discussed with patients and a decision was reached to switch to alirocumab in a reduced dosage $(75 \mathrm{mg})$ every 2 weeks. This medication was well tolerated. Two other female patients were also switched to alirocumab due to mild side effects such as muscle pain.

PCSK9 inhibition is not only a new pharmacological approach scientifically but also a new approach for patients as well. Being used to taking pills, patients must now inject their medication once or twice a month. For monitoring therapy adherence, a simple questionnaire is sent to all 38 patients (Supplemental Figure 1). Responses from 31 patients were received. Results showed not only a long individual history of the disease with an average duration of 19 years but also that nearly all patients had tried different lipid lowering drugs because of significant side effects. Over $90 \%$ of the patients had tried multiple statins and had had to switch medications in the past (Fig. 2).

Concerning therapy adherence, 27 of $31 \mathrm{pa}$ tients (87\%), that returned the anonymous questionnaire stated to have used PCSK9 inhibitors on a regular basis as prescribed. Interestingly, the remaining 4 patients reported side effects to PCSK9 inhibitors. All of these patients had previously reported side effects to statins as well (Fig. 2).

\section{Discussion}

Cardiovascular disease remains among the most common causes of death worldwide, demonstrating the need for improved treatment regimens to reduce the incidence and progression of CAD. LDL-C levels have been shown to be one of the

Table 3. Comparison of lipoprotein(a) [Lp(a)] before and after initiation of PCSK9 inhibition in 5 patients with significant elevation of $L p(a)$. Patient numbers correspond to patient from Table 1 and Table 2.

\begin{tabular}{lcccc}
\hline Patient no. & Sex & $\begin{array}{c}\text { Lp/a) before PSCK9 } \\
{[\mathrm{nmol} / \mathrm{L}(\mathrm{mg} / \mathrm{dL})]}\end{array}$ & $\begin{array}{c}\text { Lp/a) after PCSK9 } \\
{[\mathrm{nmol} / \mathrm{L}(\mathbf{m g} / \mathrm{dL})]}\end{array}$ & $\begin{array}{c}\text { Difference } \\
{[\%]}\end{array}$ \\
\hline 2 & Female & $288(120)$ & $198(83)$ & -31.25 \\
10 & Male & $312(130)$ & $259(108)$ & -16.70 \\
27 & Female & $163(68)$ & $214(89)$ & +31.30 \\
32 & Male & $255(107)$ & $189(78)$ & -25.80 \\
34 & Female & $259(108)$ & $187(77)$ & -27.80 \\
\hline
\end{tabular}




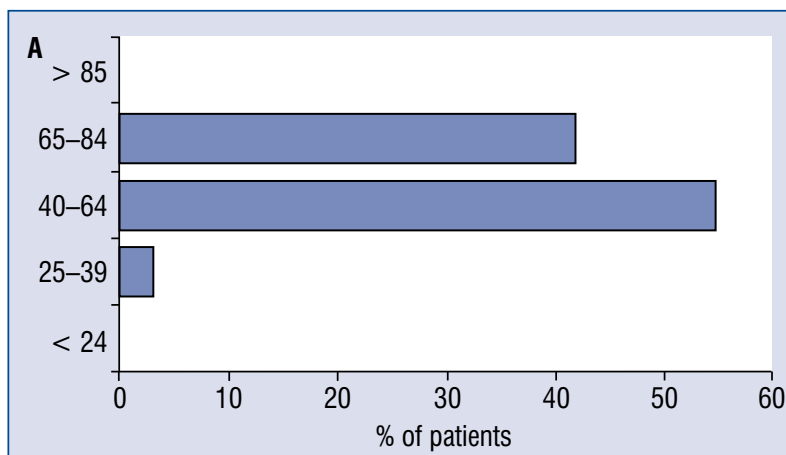

B
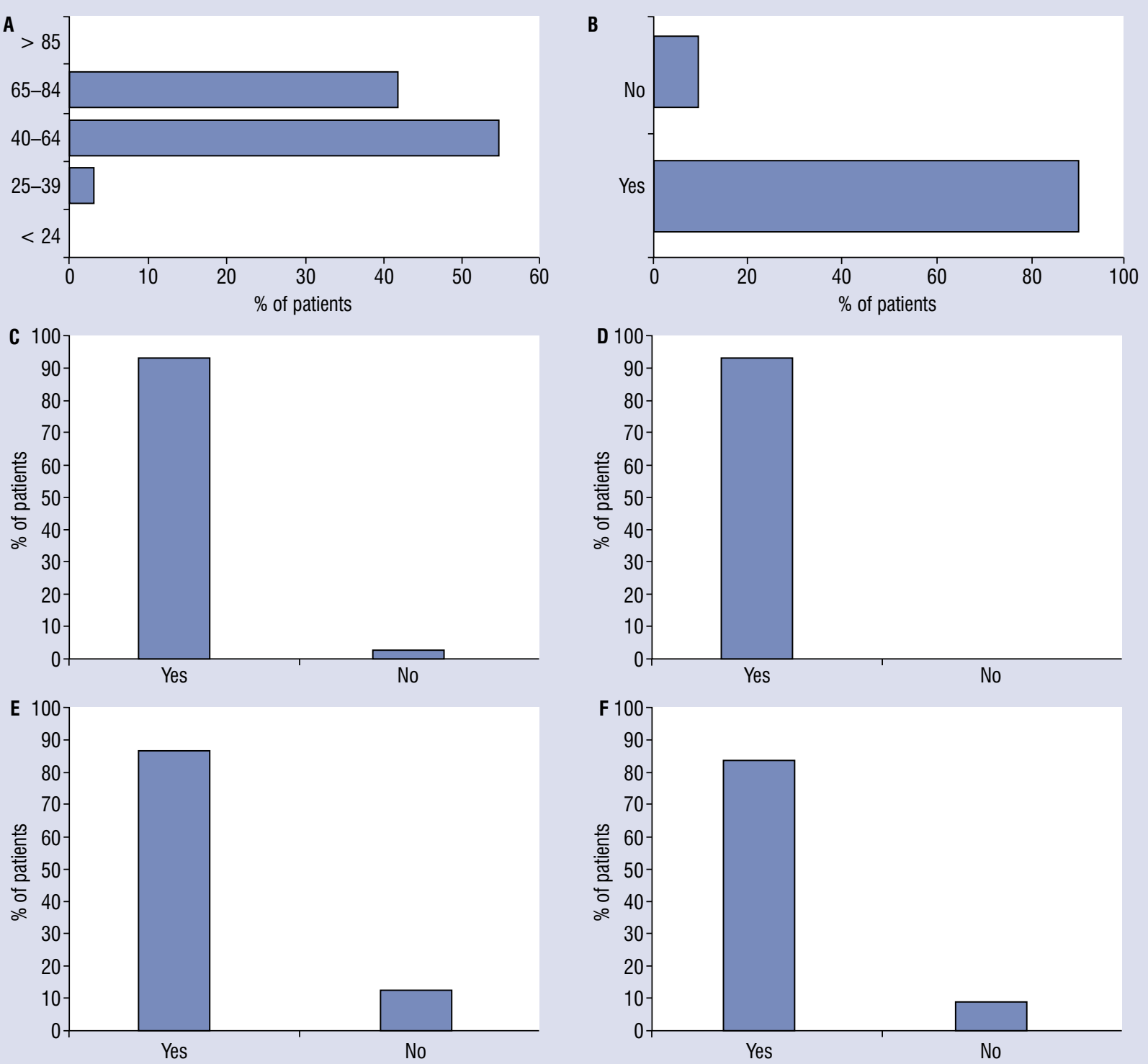
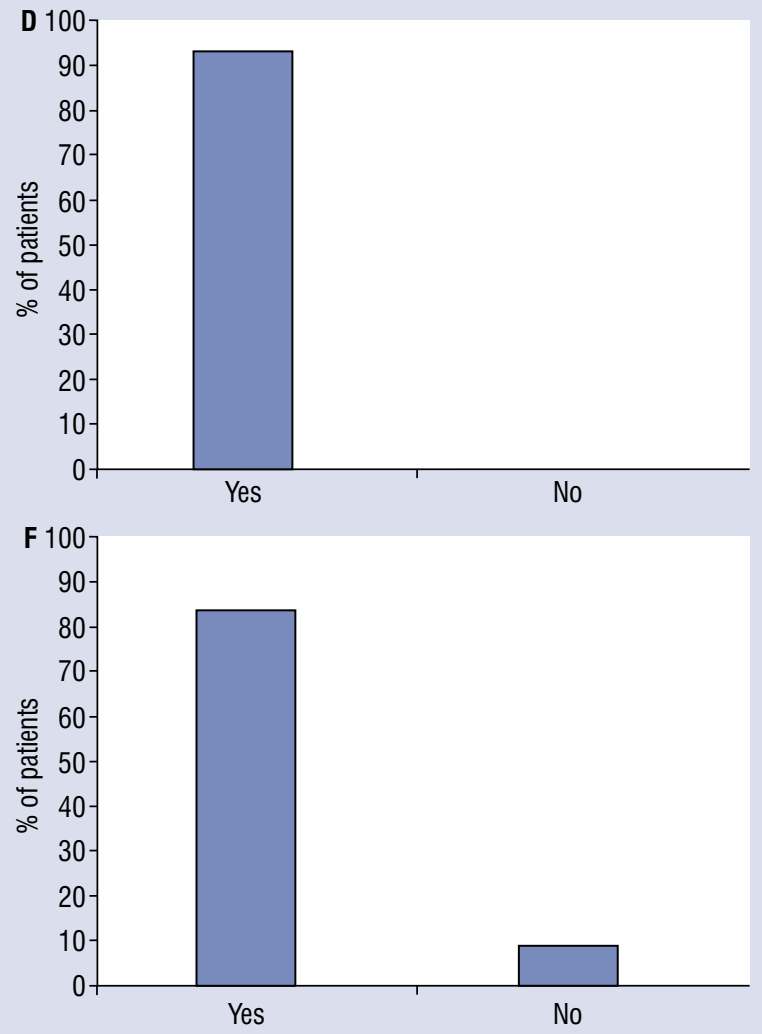

Figure 2. A questionnaire sent to all 38 patients was returned by 31 patients; A. Age of patients; B. Percent of patients that suffered from side effects of other lipid lowering drugs before PCSK9 inhibitor therapy; C. Percent of patients that needed a switch of lipid lowering therapy in the past; D. Percent of patients that knew why their physician switched the medication to a PCSK9 inhibitor; E. Percent of patients that injected the medication as prescribed; F. Percent of patients that felt safe using a PCSK9.

main therapeutic parameters in the treatment and prevention of atherosclerosis and CAD.

Herein is data reported from patients treated with PCSK9 inhibitors outside of clinical trials in a real-world cohort of 38 patients. All but two of these patients exhibited a very high-risk profile for CAD with a recommended LDL-C target range of $<70 \mathrm{mg} / \mathrm{dL}$. These patients reported that they had not only suffered from high LDL-C levels for an average of 19 years but also experienced myocardial infarction and/or other CVD. They had not been able to reach their specific LDL-C target range although their conventional therapy regimens reduced LDL-C of on average about $38 \%$. This cohort of patients had very high therapy naïve starting LDL-C levels (average $260 \mathrm{mg} / \mathrm{dL}$ ), illustrating the difficulties medical doctors face when aiming to reach LDL-C targets with conventional therapy. In particular, side effects such as muscular and articular pain frequently prevented further dose escalation. With the help of an anonymous questionnaire the study was also able to show that 
the vast majority of patients had to switch their medication of statins and ezetimibe based therapies due to recurrent side effects. Most of the patients that initially used statins had to change to less potent statins like fluvastatin during the course of the disease. In addition to the high starting, therapy-naïve LDL-C, the frequent side effects of potent statins, the failure to reach the maximum dose and switching to less potent statins played a major role in the low frequency of patients that reached the target LDL-C levels in this cohort.

After the addition of PCSK9 inhibitors, 16 of 38 patients achieved target LDL-C levels. These results illustrate not only the effectiveness of PCSK9 inhibitors but also the importance of conventional therapy and the need to maximize its effect. It is advisable to titrate the dose of statins to the maximum tolerated level. Nevertheless, the maximum allowed dosage of any prescribed statin was reached in less than half of these patients. In addition, statins should be combined with ezetimibe and colesevelam to increase LDL-C reduction prior to initiation of PCSK9 inhibitor therapy.

In the present cohort 5 patients were detected showing elevated levels of $\mathrm{Lp}(\mathrm{a})$. Four of these patients showed a reduction on average of $25 \%$ in Lp(a) levels. Surprisingly, 1 patient exhibited an increase of about $31 \%$, showing that the effects PCSK inhibition on $\mathrm{Lp}$ (a) levels are not uniform among patients.

From the questionnaires it was learned that therapy adherence to PCSK9 inhibitors was relatively high as compared to conventional therapy. Despite the fact that 6 patients reported side effects under PCSK9 inhibitor therapy, none of them had to terminate treatment and only one patient switched to a reduced dose of alirocumab. Interestingly, all patients that had side effects from PCSK9 inhibiting therapy were female. Furthermore, only $12 \%$ of total patients admitted to not injecting the inhibitor on a regular basis. Interestingly, these patients all had experienced side effects on statin based therapies before starting evolocumab or alirocumab. Therefore, to reduce the risk of incompliance due to the fear of side effects, it was suggested that patients with previous experience of side effects should be particularly well informed. In general, in-depth information about the medication and close supervision by the prescribing medical doctor is advisable.

Concerning safety, one major side effect was seen (severe hypotension with the need of hospitalization) which prompted us to change medication. Side effects, risks and benefits of this medication was discussed with our patient and a decision was made to switch to alirocumab in a reduced dosage $(75 \mathrm{mg})$ every 2 weeks. The new therapy was well tolerated.

This study emphasizes that initiation of PCSK9 inhibitor treatment should be carefully checked. Furthermore, risks and benefits of this therapy needs to be discussed in depth with patients. In addition, changing between evolocumab and alirocumab in the case of severe side effects may also be an option. The present results support ESC/EAS consensus statement [12] that suggests that the use of a PCSK9 inhibitor is worthwhile in patients with a LDL-C level $>140 \mathrm{mg} / \mathrm{dL}$ or a realistic reduction of LDL-C of about $50 \%$. Genetic testing should also be encouraged for familial hypercholesterolemia by assessing LDL receptor mutations. The present results show that the chance of detecting a mutation in this subgroup of cardiovascular patients is very high.

In summary, results document a highly efficient lowering of LDL-C with PCSK9 inhibitors in a real-world cohort. Furthermore, they document a satisfactory therapy adherence to this new medication outside of clinical trials. Nevertheless, target levels were not achieved in the majority of patients even after the addition of a PCSK9. Conventional statin-based therapy is still needed in combination with PCSK9 inhibitor therapy. Identification of individuals at highest risk of an (recurrent) event is paramount, as they will potentially benefit to the greatest degree.

\section{Conclusions}

PCSK9-inhibitors were well tolerated in a cohort of patients at high or very high cardiovascular risk and therapy adherence was overall satisfactory. Several patients who were not able to tolerate high doses of previous treatment regimens and did not reach target LDL-C levels successfully achieved recommended LDL-C treatment goals on PCSK9-inhibitors. Lowering of Lp(a) upon PCSK9 inhibition might be an additional benefit, but was not achieved in all patients. It is recommended that risks and benefits of side effects and costs be taken into account when prescribing PCSK9-inhibitors. Furthermore, switching between alirocumab and evolocumab in the case of severe side effects may be an option, but further research is needed.

\section{Acknowledgements}

We thank Dr. Inga Sörensen-Zender for critically reading this manuscript.

Conflict of interest: None declared 


\section{References}

1. Naghavi M, Wang H, Lozano R, et al. Global, regional, and national age-sex specific all-cause and cause-specific mortality for 240 causes of death, 1990-2013: a systematic analysis for the Global Burden of Disease Study 2013. Lancet. 2015; 385: 117-71.

2. Townsend N, Wilson L, Bhatnagar P, et al. Cardiovascular disease in Europe: epidemiological update 2016. Eur Heart J. 2016; 37(42): 3232-3245, doi: 10.1093/eurheartj/ehw334, indexed in Pubmed: 27523477.

3. Yusuf S, Hawken S, Ounpuu S, et al. Effect of potentially modifiable risk factors associated with myocardial infarction in 52 countries (the INTERHEART study): case-control study. Lancet. 2004; 364(9438): 937-952, doi: 10.1016/S0140-6736(04)17018-9, indexed in Pubmed: 15364185.

4. Ference BA, Yoo W, Alesh I, et al. Effect of long-term exposure to lower low-density lipoprotein cholesterol beginning early in life on the risk of coronary heart disease: a Mendelian randomization analysis. J Am Coll Cardiol. 2012; 60(25): 2631-2639, doi: 10.1016/j.jacc.2012.09.017, indexed in Pubmed: 23083789.

5. Nordestgaard BG, Chapman MJ, Humphries SE, et al. European Atherosclerosis Society Consensus Panel. Familial hypercholesterolaemia is underdiagnosed and undertreated in the general population: guidance for clinicians to prevent coronary heart disease: consensus statement of the European Atherosclerosis Society. Eur Heart J. 2013; 34(45): 3478-90a, doi: 10.1093/eurheartj/eht273, indexed in Pubmed: 23956253.

6. Fulcher J, O'Connell R, Voysey M, et al. Efficacy and safety of LDL-lowering therapy among men and women: meta-analysis of individual data from 174,000 participants in 27 randomised trials. Lancet. 2015; 385(9976): 1397-1405, doi: 10.1016/S01406736(14)61368-4, indexed in Pubmed: 25579834.

7. Cannon CP, Blazing MA, Giugliano RP, et al. IMPROVE-IT Investigators. Ezetimibe Added to Statin Therapy after Acute Coronary Syndromes. N Engl J Med. 2015; 372(25): 2387-2397, doi: 10.1056/NEJMoa1410489, indexed in Pubmed: 26039521.

8. Packard CJ, Ford I. Long-term follow-up of lipid-lowering trials. Curr Opin Lipidol. 2015; 26(6): 572-579, doi: 10.1097/ MOL.0000000000000230, indexed in Pubmed: 26780010.

9. Collins R, Reith C, Emberson J, et al. Interpretation of the evidence for the efficacy and safety of statin therapy. Lancet. 2016;
388(10059): 2532-2561, doi: 10.1016/S0140-6736(16)31357-5, indexed in Pubmed: 27616593.

10. Piepoli M, Hoes A, Agewall S, et al. 2016 European Guidelines on cardiovascular disease prevention in clinical practice. Eur Heart J. 2016; 37(29): 2315-2381, doi: 10.1093/eurheartj/ ehw106.

11. Catapano AL, Graham I, De Backer G, et al. 2016 ESC/EAS Guidelines for the Management of Dyslipidaemias. Eur Heart J. 2016; 37(39): 2999-3058, doi: 10.1093/eurheartj/ehw272, indexed in Pubmed: 27567407.

12. Landmesser U, Chapman MJ, Farnier M, et al. European Society of Cardiology (ESC), European Atherosclerosis Society (EAS). European Society of Cardiology/European Atherosclerosis Society Task Force consensus statement on proprotein convertase subtilisin/kexin type 9 inhibitors: practical guidance for use in patients at very high cardiovascular risk. Eur Heart J. 2017; 38(29): 2245-2255, doi: 10.1093/eurheartj/ehw480, indexed in Pubmed: 27789571.

13. Austin MA, Hutter CM, Zimmern RL, et al. Genetic causes of monogenic heterozygous familial hypercholesterolemia: a HuGE prevalence review. Am J Epidemiol. 2004; 160(5): 407-420, doi: 10.1093/aje/kwh236, indexed in Pubmed: 15321837.

14. Haase A, Goldberg AC. Identification of people with heterozygous familial hypercholesterolemia. Curr Opin Lipidol. 2012; 23(4): 282-289, doi: 10.1097/MOL.0b013e3283556c33, indexed in Pubmed: 22801386.

15. Apheresebehandlung bei isolierter Lp(a)-Erhöhung, Zusammenfassende Dokumentation zum Bewertungsverfahren des Unterausschusses „Ärztliche Behandlung“ des Gemeinsamen Bundesausschusses.

16. Tsimikas S. A Test in Context: Lipoprotein(a): Diagnosis, Prognosis, Controversies, and Emerging Therapies. J Am Coll Cardiol. 2017; 69(6): 692-711, doi: 10.1016/j.jacc.2016.11.042, indexed in Pubmed: 28183512.

17. Rasch D, Kubinger K, Moder K. The two-sample t test: pretesting its assumptions does not pay off. Statistical Papers. 2009; 52(1): 219-231, doi: 10.1007/s00362-009-0224-x.

18. Ruxton G. The unequal variance $t$-test is an underused alternative to Student's t-test and the Mann-Whitney U test. Behavioral Ecology. 2006; 17(4): 688-690, doi: 10.1093/beheco/ /ark016. 